\title{
Traveling fronts and wave propagation failure in an inhomogeneous neural network
}

\author{
Paul C. Bressloff \\ Department of Mathematics, University of Utah \\ Salt Lake City, Utah 84112
}

October 22, 2000

\begin{abstract}
We use averaging and homogenization theory to study the propagation of traveling wavefronts in an inhomogeneous excitable neural medium. Motivated by the functional architecture of primary visual cortex, we model the inhomogeneity as a periodic modulation in the long-range neuronal connections. We derive an expression for the effective wavespeed and show that propagation failure can occur if the speed is too slow or the degree of inhomogeneity is too large. We find that there are major qualitative differences in the wavespeed for different choices of the homogenized weight distribution.
\end{abstract}




\section{Introduction}

A common starting point for analyzing the large-scale dynamics of cortex is to treat nerve tissue as a continuous two-dimensional medium and to describe the dynamics in terms of evolution equations of the form $[1,2,3]$

$$
\tau_{0} \frac{\partial u(x, t)}{\partial t}=-u(x, t)+\int_{-\infty}^{\infty} \widehat{w}\left(x, x^{\prime}\right) f\left(u\left(x^{\prime}, t\right)\right) d x^{\prime}+I(x, t)
$$

The scalar field $u(x, t)$ represents the local activity of a population of excitatory neurons at cortical position $x \in \mathbf{R}^{2}$ at time $t, \tau_{0}$ is a time contant (associated with membrane leakage currents or synaptic currents), $I(x, t)$ is an external input, and the positive distribution $\widehat{w}\left(x, x^{\prime}\right)$ is the strength of connections from neurons at $x^{\prime}$ to neurons at $x$. [Populations of inhibitory neurons can be incorporated into equation (1.1) by taking $u$ to be a vector-valued field and replacing $w$ by a matrix of distributions with both positive and negative components]. The nonlinearity $f$ is often assumed to be a smooth monotonically increasing function of the form

$$
f(u)=\frac{1}{1+\mathrm{e}^{-\beta(u-\alpha)}}
$$

where $\beta$ is a gain parameter and $\alpha$ is a threshold. This becomes a step function in the high-gain limit $\beta \rightarrow \infty$, that is, $f(u)=\Theta(u-\alpha)$ where $\Theta(x)=1$ if $x>0$ and is zero otherwise.

A major simplification concerning the structure of cortex is to assume that it is homogeneous and isotropic, with the strength of connections depending on the (Euclidean) distance between presynaptic and postsynaptic neurons. More specifically, $\widehat{w}\left(x, x^{\prime}\right)=$ $w\left(\left|x-x^{\prime}\right|\right)$ with $w(s)$ a decreasing function of separation $s$. Under this approximation the weight distribution is invariant with respect to the Euclidean group of rigid body motions in the plane. Euclidean symmetry plays a key role in determining the types of solutions that can be generated spontaneously in such networks $[4,5]$.

Another important property of homogeneous networks is that they support the propagation of traveling waves $[6,7]$. This is of interest since a number of experimental studies have observed waves of excitation propagating in cortical slices when stimulated appropriately $[8,9]$. The propagation velocity of such waves is of order $0.06 \mathrm{~ms}^{-1}$, which is much slower than the typical speed of $0.5 \mathrm{~ms}^{-1}$ found for action potential propagation along axons. (However, it is possible that the conditions for wave propagation in the intact cortex are different since much of the long-range cortical connectivity is lost during slice preparation). From a mathematical perspective, traveling wave solutions are very sensitive to the degree of homogeneity in the connectivity pattern, particularly in the case 
of slowly moving waves. This is a consequence of the fact that traveling wave solutions are not structurally stable, that is, they correspond to heteroclinic orbits of an equivalent dynamical system. Keener has recently explored this issue within the context of reactiondiffusion equations modeling excitable chemical media [10,11]. Using a careful application of averaging or homogenization theory, he has shown that if the level of non-uniformity is sufficiently large or the wave velocity is sufficiently small then propagation failure can occur.

In this paper we extend the analysis of Keener $[10,11]$ to the case of wave propagation in inhomogeneous neural networks. Our work is motivated by the fact that although the assumption of cortical homogeneity is reasonable at the macroscopic level, there are additional structures at the microscopic level that could modify this simple picture (see section 2). For example, in the case of the primary visual cortex (V1) there are a number of functional maps superimposed on the underlying retinotopic representation of the visual field $[12,13]$. These maps, which correspond to additional cortical labels such as ocular dominance and orientation preference, introduce an approximate periodic tiling of the cortical plane whose wavelength is comparable to a single hyperecolumn spacing of around $1 \mathrm{~mm}$ (in cats and monkeys). It is likely that such functional periodicity is associated with a corresponding periodicity in the anatomy. Within the framework of large-scale cortical models (1.1), the periodic microstructure of the cortex can be incorporated by taking a weight distribution ${ }^{1}$

$$
\widehat{w}\left(x, x^{\prime}\right)=w\left(\left|x-x^{\prime}\right|\right) h\left(\left|x-x^{\prime}\right| / \epsilon\right) k\left(x^{\prime} / \epsilon\right)
$$

where $h, k$ are $2 \pi$-periodic functions and $\epsilon$ determines the microscopic length-scale. If $k=1$ then the cortex still acts like a homogeneous medium with the effective connection strength a periodically modulated function of spatial separation. On the other hand, if there are inhomogeneities arising from the microscopic level then these will result in a periodically heterogeneous medium for which $k \neq 1$. Absorbing the factor $h$ into $w$ and substituting into equation (1.1) gives the inhomogeneous evolution equation (for zero external inputs)

$$
\tau_{0} \frac{\partial u(x, t)}{\partial t}=-u(x, t)+\int_{-\infty}^{\infty} w\left(\left|x-x^{\prime}\right|\right) k\left(x^{\prime} / \epsilon\right) f\left(u\left(x^{\prime}, t\right)\right) d x^{\prime}
$$

\footnotetext{
${ }^{1} \mathrm{~A}$ more detailed analysis would require the introduction of additional cortical labels such as orientation preference $\phi \in S^{1}$ and considering a generalized evolution equation for the activity variable $a(x, \phi, t)$. Bressloff et al [5] have developed a model of V1 along these lines by considering a weight distribution $w\left(x, \phi ; x^{\prime}, \phi,\right)$ that is homogeneous but anisotropic with respect to cortical cordinates. However, Euclidean invaraince is preserved with respect to a group action on the extended space $\mathbf{R}^{2} \times S^{1}$.
} 
The main goal of this paper is to determine the behavior of propagating wavefronts in a one-dimensional version of equation (1.4) by extending the averaging method of Keener $[10,11]$ to the case of integro-differential equations. This generates an analytical expression for the wavespeed and waveform shape, from which it can be deduced that propagation failure occurs when the medium is sufficiently inhomogeneous (see section 3). An explicit calculation of the wavespeed is then carried out in the case of piecewise linear dynamics (see section 4). We show that the $\epsilon$-dependence of solutions is sensitive to the choice of weight distribution $w$. In particular, there are major qualitative differences between exponential and Gaussian weight distributions due to the fact that in the latter case there are transcendentally small (non-perturbative) $\epsilon$-dependent contributions to the wavespeed. Interestingly, such terms only occur for smooth nonlinearities in the models considered by Keener [10, 11].

\section{Is the cortex a homogeneous medium?}

The most basic feature of the functional architecture of V1 is an orderly retinotopic mapping of the visual field onto the surface of cortex, with the left and right halves of visual field mapped onto the right and left cortices respectively. Superimposed upon this retinotopic map are a number of additional functional maps that each have an approximately periodic structure [13].

(a) Ocular dominance. Cells tend to respond more strongly to stimuli presented in one eye than in the other, and are said to exhibit ocular dominance. Cells sharing the same ocular dominance are grouped together into non-overlapping regions that form an alternating pattern of right and left eye preference across V1. Such regions have a characteristic periodicity and morphology that is species-dependent. For example, in the macaque monkey ocular dominace regions consist of branching stripes that have an approximately uniform width of $0.4 \mathrm{~mm}[14]$.

(b) Orientation columns. Most cells in V1 respond optimally to a bar or edge moving at an appropriate orientation and velocity in its receptive field [15, 16]. Cells of similar orientation preference are organized into vertical columns whereas there is a systematic variation of orientation preference transversely across the cortex [17]. In particular, when a micro-electrode is moved past a series of columns the preferred orientation rotates at an approximately constant rate over a distance of around $1 \mathrm{~mm}$, although discontinuous jumps of up to $90^{\circ}$ are also observed. A much more detailed picture of the two-dimensional 
arrangement of orientation preferences has recently been revealed using optical imaging $[18,19,20]$. Optical imaging detects neural activity with a sensitive camera, either by applying a voltage-sensitive dye to the surface of the cortex or by recording small changes in the absorbance properties of neural tissue that is correlated with activity. The basic topography revealed by this method has a number of characteristic features [12] as illustrated schematically in figure 1.

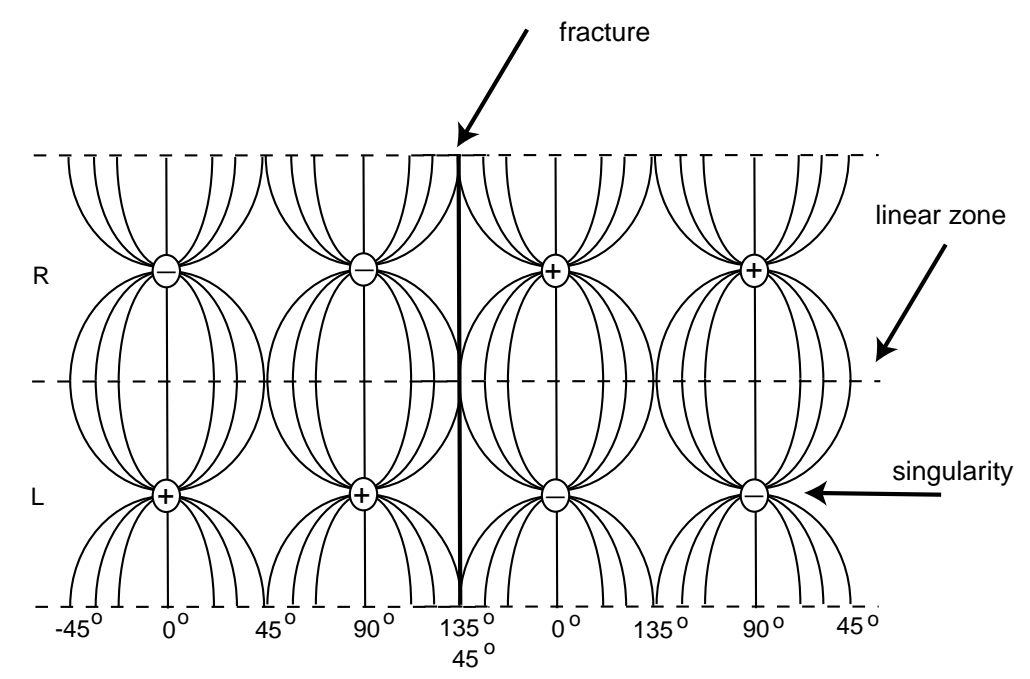

Figure 1: Schematic illustration of orientation contours, singularities and linear zones in V1 as revealed by optical imaging. The actual cortex is not as ordered as the figure.

(i) Orientation preference changes continuously as a function of cortical location except at singularities (or pinwheels). There is an approximately equal number of positive and negative singularities that are distinguished according to whether orientation preference rotates anticlockwise or clockwise around the singularity.

(ii) There exist linear regions, approximately $800 \times 800 \mu m^{2}$ in area (in macaque monkeys), bounded by singularities, within which iso-orientation regions form parallel slabs.

(iii) Orientation changes more slowly in the regions between two singularities of the same polarity (saddle points) but changes abruptly between singularities of opposite sign (along line fractures).

(iv) Iso-orientation slabs tend to cross the borders of ocular dominance stripes at right angles. Singularities tend to align with the centers of ocular dominance stripes. 
(c) Cytochrome Oxidase (CO) blobs. Single electrode recordings across monkey visual cortex have revealed that there are regions in the upper layers of V1 in which orientation selectivity of cells is relatively poor or even absent [21]. These regions, which are about $0.2 \mathrm{~mm}$ in diameter and about $0.4 \mathrm{~mm}$ apart, were found to coincide with the patchy dark spots or blobs revealed under staining of $\mathrm{V} 1$ with the enzyme cytochrome oxidase $(\mathrm{CO})$. The blob regions coincide with cells that are more metabolically active and hence richer in their levels of CO. They are located in the centers of ocular dominance stripes, which indicates some association with orientation singularities. It has also been suggested that the CO blobs could be the sites of functionally and anatomically distinct channels of visual processing. For example, there is some evidence that they respond preferentially to low spatial frequencies [22] and are selective to coloured stimuli [21]. However, these observations are still controversial [23].

The above experimental findings suggest that there is an underlying periodicity in the microstructure of V1 with a period of approximately $1 \mathrm{~mm}$ (in cats and primates). The fundamental domain of this periodic tiling of the cortical plane is the hypercolumn [24], which contains two sets of all iso-orientation patches in the range $0 \leq \phi<\pi$, one for each eye, and four singularities as shown in figure 1. The next step is to determine how this periodic structure manifests itself anatomically? Two cortical circuits have been fairly well characterized. There is a local circuit operating at sub-hypercolumn dimensions which comprises strong orientation specific recurrent excitation and weaker intra-hypercolumnar inhibition [25, 26]. The other circuit operates between hypercolumns, connecting cells with similar orientation preferences separated by several millimetres of cortical tissue $[27,28,29,30,31,32]$. The intrinsic lateral or horizontal connections that mediate this circuit arise almost exclusively from excitatory neurons [33, 34], although $20 \%$ terminate on inhibitory cells and can thus have significant inhibitory effects [35].

It appears that the horizontal connections also tend to link cells that are similar with respect to other cortical labels [31]. This observation provides a possible mechanism for the periodic weight modulation assumed in the model described by equation (1.4). For if there is an asymmetry in the strength of connections blobs $\rightarrow$ blobs relative to interblobs $\rightarrow$ interblobs then this would result in a periodic modulation of the long-range connections. A similar comment holds if there is an asymmetry between pinwheels $\rightarrow$ pinwheels and linearzones $\rightarrow$ linearzones. Moreover, since blobs are separated by around $0.4 \mathrm{~mm}$ whereas the horizontal connections can extend up to $6 \mathrm{~mm}$ [28], it follows that the periodicity of the heterogeneity could be an order of magnitude smaller than the correlation length of the long-range connections. As previously mentioned, there is still a debate concerning whether or not there really are functional and anatomical differences 
between, for example, blobs and interblobs. However, as our analysis in this paper will show, even a small heterogeneity can have a dramatic effect on the dynamical properties of an excitable neural medium. In particular, it can lead to wave propagation failure.

\section{Averaging theory and homogenization}

In this section we analyze wavefront progation and its failure in a one-dimensional inhomogeneous network evolving according to equation (1.4). It is convenient to set $k(x / \epsilon)=$ $1+A^{\prime}(x / \epsilon)$ where $A^{\prime}$ is a $2 \pi$-periodic function and $x \in \mathbf{R}$ so that the evolution equation takes the form (for $\tau_{0}=1$ )

$$
\frac{\partial u(x, t)}{\partial t}=-u(x, t)+\int_{-\infty}^{\infty} w\left(x-x^{\prime}\right)\left[1+A^{\prime}\left(x^{\prime} / \epsilon\right)\right] f\left(u\left(x^{\prime}, t\right)\right) d x^{\prime}
$$

We shall assume that $\epsilon$ is a small parameter such that the periodic modulation occurs on a smaller length scale than the correlation length of $w(x)$. A zeroth order approximation of equation (3.1), which is obtained by peforming a spatial averaging with respect to the periodic weight modulation, is the homogeneous equation

$$
\frac{\partial u(x, t)}{\partial t}=-u(x, t)+\int_{-\infty}^{\infty} w\left(x-x^{\prime}\right) f\left(u\left(x^{\prime}, t\right)\right) d x^{\prime}
$$

It can be shown that equation (3.2) has a unique propagating wavefront solution $u(x, t)=$ $U(x-c t)$, where $c$ is the wave speed, provided that the following conditions hold [6]:

(i). The nonlinear function $f$ is continuously differentiable and $f^{\prime}>0$ (monotonically increasing function of $u$ ).

(ii). The function $-u+f(u)$ has exactly three zeros with $u_{0}<u_{1}<u_{2}$ and $f^{\prime}\left(u_{0}\right)<1$, $f^{\prime}\left(u_{2}\right)<1$. Thus $u(x)=u_{0,2}$ are stable fixed point solutions of $(3.2)$.

(iii). $w$ is an absolutely continuous, even, positive function with $\int_{-\infty}^{\infty} w(x) d x<\infty$

Conditions (i) and (ii) apply for example to the function (1.2) over a range of values of $\beta$ and $\alpha$. Although the step function $f(x)=\Theta(x-\alpha)$ is not itself continuously differentiable, the wavefront solution can be calculated explicitly in this limiting case $[6,7]$. We shall assume that $w$ satisfies condition (iii).

We would like to determine whether or not the inhomogeneous network given by equation (3.1) also supports wave propagation. We shall proceed by following the averaging approach of Keener [10], who studied the related problem of wave front propagation in 
excitable chemical media with discrete release sites. In particular, Keener considered a simplified model of calcium release and uptake in cardiac cells given by

$$
\frac{\partial u}{\partial t}=\frac{\partial^{2} u}{\partial x^{2}}-u+\left[1+A^{\prime}(x / \epsilon)\right] f(u)
$$

with $u$ representing calcium concentration and $A^{\prime}(x)$ representing the local density of calcium release sites. (See also the analysis of bistable equations with inhomogeneous resistivities [11]). He showed that an inhomogeneous medium can have very different properties from the averaged homogeneous medium, namely, the former can exhibit propagation failure. Moreover, he explored this difference within the context of the averaging theorem [36, 37]. The averaging theorem applies to systems of differential equations of the form

$$
\frac{d u}{d x}=F\left(u, \frac{x}{\epsilon}, \epsilon\right)
$$

and states that for sufficiently small $\epsilon$ there exists an exact change of variables $u=$ $y+Y(y, x, \epsilon)$ that transforms the system of equations into

$$
\frac{d y}{d x}=F_{0}(y)+\epsilon F_{1}\left(y, \frac{x}{\epsilon}, \epsilon\right)
$$

where $F_{0}$ is the averaged function

$$
F_{0}(y)=\lim _{X \rightarrow \infty} \frac{1}{2 X} \int_{-X}^{X} F(y, x, 0) d x
$$

An important issue concerns the conditions under which trajectories of the first-order averaged or homogenized system $d y / d x=F_{0}(y)$ remain sufficiently close to trajectories of the exact system (with respect to an appropriate metric). It turns out that the crucial requirement is that the solutions of the averaged system are structurally stable [36]. However, as highlighted by Keener [10, 11], traveling fronts correspond to heteroclinic trajectories within a dynamical systems framework and are thus not structurally stable. Therefore one has to go beyond lowest order averaging in order to resolve the differences between the homogeneous and inhomogeneous systems.

It is a simple matter to see why similar considerations are expected to hold for the neural network model of equation (3.1). For suppose that the weight distribution $w$ is taken to be the exponential function

$$
w(x)=\frac{\mathrm{e}^{-|x| / \sigma}}{2 \sigma}
$$

The integro-differential equation (3.1) is then equivalent to a reaction-diffusion system (with $\tau_{0}=1, \sigma=1$ ) of the form

$$
\frac{\partial u}{\partial t}=-u+v
$$




$$
0=\frac{\partial^{2} v}{\partial x^{2}}-v+\left[1+A^{\prime}(x / \epsilon)\right] f(u)
$$

which is similar in structure to the model equation (3.3) considered by Keener [10]. However, rather than restricting ourselves to a particular weight distribution and applying the averaging method to the differential equations (3.5) and (3.6), we shall develop a version that is directly applicable to the integro-differential equation (3.1) for general $w$.

The first step is to perform an integration by parts in equation (3.1) so that

$$
\begin{aligned}
\frac{\partial u(x, t)}{\partial t}= & -u(x, t)+\int_{-\infty}^{\infty} w\left(x-x^{\prime}\right) f\left(u\left(x^{\prime}, t\right)\right) d x^{\prime} \\
& +\epsilon \int_{-\infty}^{\infty} A\left(x^{\prime} / \epsilon\right)\left[w^{\prime}\left(x-x^{\prime}\right) f\left(u\left(x^{\prime}, t\right)\right)-w\left(x-x^{\prime}\right) \frac{\partial f\left(u\left(x^{\prime}, t\right)\right)}{\partial x^{\prime}}\right] d x^{\prime}
\end{aligned}
$$

Since the inhomogeneous system is not translationally invariant it cannot have exact traveling wave solutions of the form $U(x-c t)$. Nevertheless, motivated by the structure of such solutions we perform the change of variables $\xi=x-\phi(t)$ and $\tau=t$ [10]. Equation (3.7) becomes

$$
\begin{aligned}
\frac{\partial u(\xi, \tau)}{\partial \tau}= & -u(\xi, \tau)+\int_{-\infty}^{\infty} w\left(\xi-\xi^{\prime}\right) f\left(u\left(\xi^{\prime}, \tau\right)\right) d \xi^{\prime}+\phi^{\prime} \frac{\partial u(\xi, \tau)}{\partial \xi} \\
& +\epsilon \int_{-\infty}^{\infty} A\left(\left[\xi^{\prime}+\phi\right] / \epsilon\right)\left[w^{\prime}\left(\xi-\xi^{\prime}\right) f\left(u\left(\xi^{\prime}, \tau\right)\right)-w\left(\xi-\xi^{\prime}\right) \frac{\partial f\left(u\left(\xi^{\prime}, \tau\right)\right)}{\partial \xi^{\prime}}\right] d \xi^{\prime}
\end{aligned}
$$

Next perform the perturbation expansions

$$
\begin{gathered}
u(\xi, \tau)=U(\xi)+\epsilon u_{1}(\xi, \tau)+\epsilon^{2} u_{2}(\xi, \tau)+\ldots \\
\phi^{\prime}(\tau)=c+\epsilon \phi_{1}^{\prime}(\tau)
\end{gathered}
$$

where $U(\xi)$ is the unique traveling wave solution of the corresponding homogeneous equation (3.2), that is,

$$
-c U^{\prime}(\xi)=-U(\xi)+\int_{-\infty}^{\infty} w\left(\xi-\xi^{\prime}\right) f\left(U\left(\xi^{\prime}\right)\right) d \xi^{\prime}
$$

where $c$ is the speed of the unperturbed wave. The first-order term $u_{1}$ satisfies the inhomogeneous linear equation

$$
-\frac{\partial u_{1}(\xi, \tau)}{\partial \tau}+\mathcal{L} u_{1}(\xi, \tau)=-\phi_{1}^{\prime}(\tau) U^{\prime}(\xi)+h_{1}(\xi, \phi / \epsilon)
$$

where

$$
\mathcal{L} u(\xi)=c \frac{d u(\xi)}{d \xi}-u(\xi)+\int_{-\infty}^{\infty} w\left(\xi-\xi^{\prime}\right) f^{\prime}\left(U\left(\xi^{\prime}\right)\right) u\left(\xi^{\prime}\right) d \xi^{\prime}
$$


and

$$
h_{1}(\xi, \phi / \epsilon)=-\int_{-\infty}^{\infty} A\left(\left[\xi^{\prime}+\phi\right] / \epsilon\right)\left[w^{\prime}\left(\xi-\xi^{\prime}\right) f\left(U\left(\xi^{\prime}\right)\right)-w\left(\xi-\xi^{\prime}\right) \frac{d f\left(U\left(\xi^{\prime}\right)\right)}{d \xi^{\prime}}\right] d \xi^{\prime}
$$

The linear operator $\mathcal{L}$ has a one-dimensional null-space spanned by $U^{\prime}$. The existence of $U^{\prime}$ as a null-vector follows immediately from differentiating both sides of equation (3.11), whereas its uniqueness can be shown using properties of positive linear operators [6]. Therefore, a bounded solution of equation (3.12) with respect to $\xi$ and $\tau$ will only exist if the right-hand side of equation (3.12) is orthogonal to all elements of the null-space of the adjoint operator $\mathcal{L}^{*}$. The latter is defined with respect to the inner product

$$
\int_{-\infty}^{\infty} u(\xi) \mathcal{L} v(\xi) d \xi=\int_{-\infty}^{\infty}\left[\mathcal{L}^{*} u(\xi)\right] v(\xi) d \xi
$$

where $u(\xi)$ and $v(\xi)$ are arbitrary integrable functions. Hence,

$$
\mathcal{L}^{*} u(\xi)=-c \frac{d u(\xi)}{d \xi}-u(\xi)+f^{\prime}(U(\xi)) \int_{-\infty}^{\infty} w\left(\xi-\xi^{\prime}\right) u\left(\xi^{\prime}\right) d \xi^{\prime}
$$

It can be proven that $\mathcal{L}^{*}$ also has a one-dimensional null-space [6], that is, it is spanned by some function $V(\xi)$. Equation (3.12) thus has a bounded solution if and only if

$$
K \phi_{1}^{\prime}(\tau)=\int_{-\infty}^{\infty} V(\xi) h_{1}(\xi, \phi / \epsilon) d \xi
$$

where

$$
K=\int_{-\infty}^{\infty} V(\xi) U^{\prime}(\xi) d \xi
$$

Note that $K$ is strictly positive since $V$ and $U^{\prime}$ can be chosen to have the same sign [6]. Substituting for $h$ using equations (3.14) and (3.10) and performing an integration by parts leads to a differential equation for the phase $\phi$ :

$$
\frac{d \phi}{d \tau}=c+\epsilon \Phi_{1}\left(\frac{\phi}{\epsilon}\right)
$$

where

$$
\Phi_{1}\left(\frac{\phi}{\epsilon}\right)=\frac{1}{K} \int_{-\infty}^{\infty} \int_{-\infty}^{\infty} w\left(\xi-\xi^{\prime}\right) A\left(\frac{\xi^{\prime}+\phi}{\epsilon}\right)\left[V^{\prime}(\xi) f\left(U\left(\xi^{\prime}\right)\right)+V(\xi) \frac{d f\left(U\left(\xi^{\prime}\right)\right)}{d \xi^{\prime}}\right] d \xi^{\prime} d \xi
$$

The phase equation (3.19) is analogous to the one derived by Keener for a reactiondiffusion model of calcium waves [10]. It implies that there are two distinct types of behavior. If the right-hand side of equation (3.19) is strictly positive then there exists a 
traveling front of the approximate form $U(x-\phi(t))$ and the average speed of propagation is

$$
\bar{c}=\frac{2 \pi \epsilon}{T}
$$

where

$$
T=\int_{0}^{2 \pi \epsilon} \frac{d \phi}{c+\epsilon \Phi_{1}\left(\frac{\phi}{\epsilon}\right)}
$$

On the other hand, if the right-hand side of equation (3.19) vanishes for some $\phi$ then there is wave propagation failure.

\section{Calculation of average wavespeed}

In this section we calculate the average wavespeed and the conditions for wave propagation failure in the special case of the step-function nonlinearity $f(u)=\Theta(u-\alpha)$. Although $f$ is not itself continuously differentiable, it can be obtained as the high-gain limit $\beta \rightarrow \infty$ of the smooth function (1.2). The advantage of using a threshold nonlinearity is that all calculations can be carried out explicitly. Moreover, when $\beta$ is finite it is necessary to develop the perturbation expansion of equation (3.8) to $\mathcal{O}\left(\epsilon^{2}\right)$ rather than $\mathcal{O}(\epsilon)$ (see section 4.3).

\subsection{Piecewise linear dynamics}

The first step in the analysis of the average wavespeed is to determine the solution $U(\xi)$ of equation (3.11) and the solution $V(\xi)$ of the adjoint linear equation $\mathcal{L}^{*} V=0$. The wave profile $U(\xi)$ in the case of the threshold nonlinearity $f(U)=\Theta(U-\alpha)$ can be obtained as follows $[6,7]$. Since the wave of the homogeneous equation is translation invariant we impose the normalization condition $U(0)=\alpha$. For concreteness let $0<\alpha<1 / 2$ and take $U(\xi)$ to be a monotonically decreasing function of $\xi$ so that $c>0, U(\xi)<\alpha$ for $\xi>0$ and $U(\xi)>\alpha$ for $\xi<0$. Equation (3.11) then reduces to

$$
-c U^{\prime}(\xi)+U(\xi)=\int_{-\infty}^{0} w\left(\xi-\xi^{\prime}\right) d \xi^{\prime}=\int_{\xi}^{\infty} w(x) d x \equiv G(\xi)
$$

which has the solution

$$
U(\xi)=\mathrm{e}^{\xi / c}\left[\alpha-\frac{1}{c} \int_{0}^{\xi} \mathrm{e}^{-y / c} G(y) d y\right]
$$


Requiring the solution to remain bounded as $\xi \rightarrow \infty$ implies that $c$ must satisfy the condition

$$
\alpha=\frac{1}{c} \int_{0}^{\infty} \mathrm{e}^{-y / c} G(y) d y=\int_{0}^{\infty}\left[1-\mathrm{e}^{-y / c}\right] w(y) d y
$$

Substitution of (4.3) into (4.2) yields the result [6]

$$
U(\xi)=\frac{1}{c} \int_{0}^{\infty} \mathrm{e}^{-y / c} G(y+\xi) d y
$$

Furthermore, differentiating both sides of equation (4.4) and using the definition of $G(\xi)$ gives

$$
U^{\prime}(\xi)=-\frac{1}{c} \int_{0}^{\infty} \mathrm{e}^{-y / c} w(y+\xi) d y \equiv-\frac{\widehat{G}(\xi)}{c}
$$

Substituting $f(U)=\Theta(U-\alpha)$ into equation (3.16) implies that $V(\xi)$ satisfies the equation

$$
c V^{\prime}(\xi)+V(\xi)=-\frac{\delta(\xi)}{U^{\prime}(0)} \int_{-\infty}^{\infty} w\left(\xi^{\prime}\right) V\left(\xi^{\prime}\right) d \xi^{\prime}
$$

This can be integrated to give

$$
V(\xi)=-\Theta(\xi) \mathrm{e}^{-\xi / c}
$$

with

$$
c U^{\prime}(0)=\int_{-\infty}^{\infty} w(\xi) V(\xi) d \xi
$$

Equations (4.7) and (4.8) are consistent since they imply

$$
U^{\prime}(0)=-\frac{1}{c} \int_{0}^{\infty} \mathrm{e}^{-y / c} w(y) d y
$$

which recovers equation (4.5) for $\xi=0$. It follows from equation (4.7) that equation (3.20) reduces to the form

$$
\begin{aligned}
& K \Phi_{1}\left(\frac{\phi}{\epsilon}\right) \\
& =\int_{-\infty}^{\infty} \int_{-\infty}^{\infty} w\left(\xi-\xi^{\prime}\right) A\left(\frac{\xi^{\prime}+\phi}{\epsilon}\right) \mathrm{e}^{-\xi / c}\left[\left(\frac{\Theta(\xi)}{c}-\delta(\xi)\right) \Theta\left(-\xi^{\prime}\right)+\Theta(\xi) \delta\left(\xi^{\prime}\right)\right] d \xi^{\prime} d \xi \\
& =\widehat{G}(0) A\left(\frac{\phi}{\epsilon}\right)+\int_{0}^{\infty} A\left(\frac{\phi-\xi}{\epsilon}\right)\left[\frac{\widehat{G}(\xi)}{c}-w(\xi)\right] d \xi
\end{aligned}
$$

Furthermore, substituting equations (4.4) and (4.7) into (3.18) shows that

$$
K=\frac{1}{c} \int_{0}^{\infty} \mathrm{e}^{-\xi / c} \widehat{G}(\xi) d \xi
$$


Equation (4.10) implies that

$$
\Phi_{1}\left(\frac{\phi}{\epsilon}\right)=\frac{\widehat{G}(0)}{K} A\left(\frac{\phi}{\epsilon}\right)+\epsilon F\left(\frac{\phi}{\epsilon}, \epsilon\right)
$$

Keeping only the lowest order contribution to $\Phi_{1}$, equation (3.22) reduces to

$$
T=\int_{0}^{2 \pi \epsilon} \frac{d \phi}{c+\epsilon \Gamma(c) A\left(\frac{\phi}{\epsilon}\right)}
$$

with $\Gamma(c)=\widehat{G}(0) / K$. It remains to specify the weight distribution $w$ (which determines the factor $\Gamma(c))$ and the periodic modulation represented by the function $A$. For the purposes of analytical tractability we shall take $A$ to be a pure sinusoid. (However, this particular choice of $A$ is not essential for the basic phenomena described below). Setting $A(x)=a \sin (x)$ in equation (4.13) we find that

$$
T=\frac{2 \pi \epsilon}{\sqrt{c^{2}-\epsilon^{2} a^{2} \Gamma(c)^{2}}}
$$

and hence

$$
\bar{c}=\sqrt{c^{2}-\epsilon^{2} a^{2} \Gamma(c)^{2}}
$$

This establishes that a sinusoidally varying heterogeneous neural medium only supports a propagating wave if the velocity $c$ of the (unique) solution of the corresponding homogeneous medium satisfies the inequality

$$
c \geq \epsilon a \Gamma(c)
$$

Since $\Gamma(0)=1$ it follows that in the case of slowly moving waves (small $c$ ), propagation failure occurs if $\epsilon a>c$, that is, if either the unperturbed wave is too slow or the nonuniformity of the medium is too large (as characterized by the wavelength $\epsilon$ or amplitude $a$ of the periodic modulation). For faster waves the critical period $\epsilon_{0}$ for wave propagation failure is no longer linear in $c$ due to the presence of the factor $\Gamma(c)$. The latter depends on the particular weight distribution $w$ as we now illustrate.

(a) Exponential weight distribution. In the case of the exponential distribution (3.4) with $\sigma=1$ (which fixes the length-scale) we find that

$$
\widehat{G}(\xi)=\frac{1}{2} \int_{0}^{\infty} \mathrm{e}^{-y / c} \mathrm{e}^{-(y+\xi)} d y=\frac{c}{2(c+1)} \mathrm{e}^{-\xi}
$$

for $\xi \geq 0$ and

$$
K=\frac{c}{2(c+1)^{2}}
$$


so that

$$
\Gamma(c)=1+c
$$

The resulting average wavespeed (4.15) is plotted as a function of $\epsilon$ and various unperturbed speeds $c$ in figure 2(a). The corresponding critical period $\epsilon_{0}$ for propagation failure is shown in figure 3(a). It can be seen that the linear relationship between $c$ and $\epsilon_{0}$ breaks down for large $c$ with $a \epsilon_{0} \rightarrow 1$ as $c \rightarrow \infty$. On the other hand, $\epsilon_{0}$ is a linear function of the threshold $\alpha$ over the range $0<\alpha<1 / 2$, see figure 3(b). In particular, from equation (4.2) we find that

$$
U(\xi)=\left\{\begin{array}{cc}
\frac{1}{2(c+1)} \mathrm{e}^{-\xi} & \text { for } \xi>0 \\
1+(\alpha-1) \mathrm{e}^{\xi / c}+\frac{1}{2(c-1)}\left[\mathrm{e}^{\xi}-\mathrm{e}^{\xi / c}\right] & \text { for } \xi<0
\end{array}\right.
$$

with

$$
\alpha=\frac{1}{2(c+1)}
$$

Thus wave propagation failure will occur when the threshold satisfies the inequality

$$
\frac{1}{2}>\alpha>\frac{1}{2}-\frac{\epsilon a}{2}>0
$$
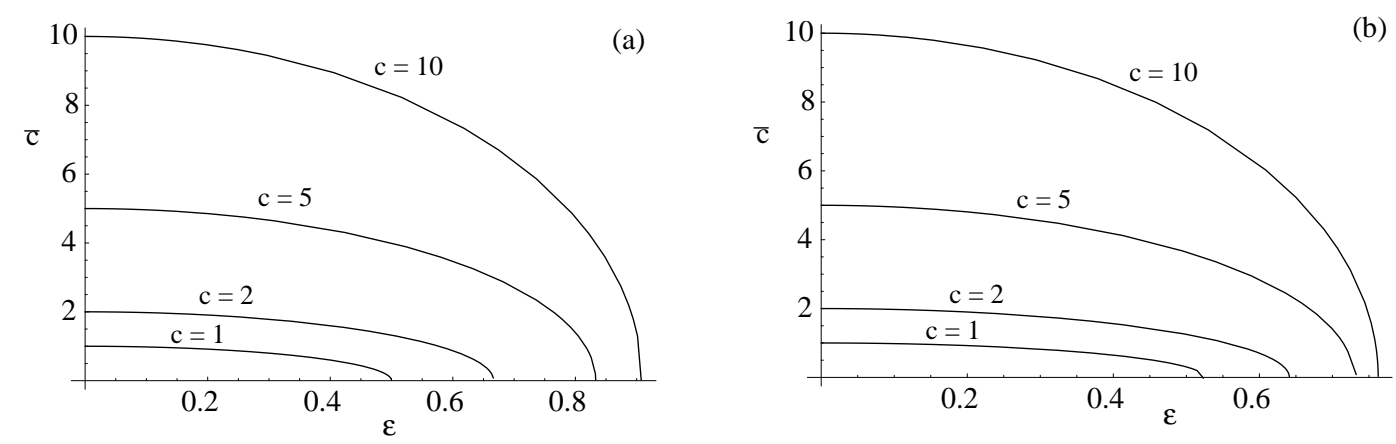

Figure 2: Average wavespeed $\bar{c}$ satisfying equation (4.15) is plotted as a function of $\epsilon$ for a sinusoidal modulation of unit amplitude. (a) Exponential weight distribution and (b) Gaussian weight distribution.

(b) Gaussian weight distribution. In the case of the Gaussian distribution

$$
w(x)=\frac{1}{\sqrt{2 \pi \sigma^{2}}} \mathrm{e}^{-x^{2} / 2 \sigma^{2}}
$$



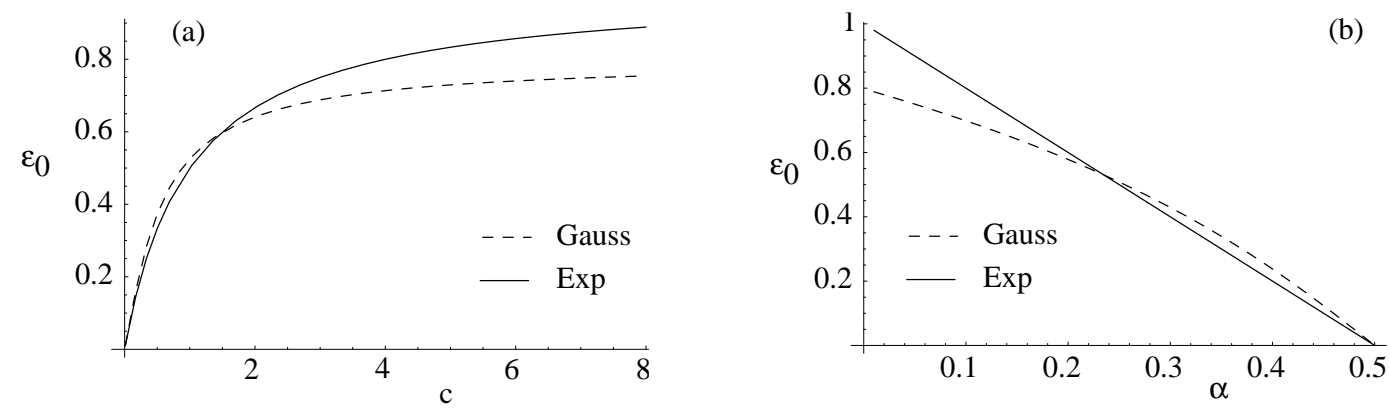

Figure 3: Critical period $\epsilon_{0}$ for wave propagation failure is plotted as a function of (a) unperturbed wavespeed $c$ and (b) threshold $\alpha$ for an exponential weight distribution (solid line) and a Gaussian weight distribution (dashed line).

(with $\sigma=1$ ) we find that

$$
\begin{aligned}
\widehat{G}(\xi) & =\frac{1}{\sqrt{2 \pi}} \int_{0}^{\infty} \mathrm{e}^{-y / c} \mathrm{e}^{-(y+\xi)^{2} / 2} d y \\
& =\frac{1}{2} \exp \left(\frac{\xi}{c}+\frac{1}{2 c^{2}}\right) \operatorname{erfc}\left[\frac{\xi+c^{-1}}{\sqrt{2}}\right]
\end{aligned}
$$

where $\operatorname{erfc}(x)$ is the complementary error function

$$
\operatorname{erfc}(x)=\frac{2}{\sqrt{\pi}} \int_{x}^{\infty} \mathrm{e}^{-y^{2}} d y
$$

Furthermore, using the result

$$
\int_{0}^{\infty} \operatorname{erfc}(x+y) d x=\frac{1}{\sqrt{\pi}} \mathrm{e}^{-y^{2}}-y \operatorname{erfc}(y)
$$

we have

$$
K=\frac{1}{2 c} \sqrt{\frac{2}{\pi}}-\frac{1}{2 c^{2}} \exp \left(\frac{1}{2 c^{2}}\right) \operatorname{erfc}(1 / \sqrt{2} c)
$$

so that

$$
\Gamma(c)=\frac{c^{2} \operatorname{erfc}(1 / \sqrt{2} c)}{c \sqrt{\frac{2}{\pi}} \exp \left(-\frac{1}{2 c^{2}}\right)-\operatorname{erfc}(1 / \sqrt{2} c)}
$$

In the slow velocity regime we can use the asymptotic expansion

$$
\operatorname{erfc}(x)=\frac{\mathrm{e}^{-x^{2}}}{\sqrt{\pi} x}\left[1-\frac{1}{2 x^{2}}+\frac{3}{4 x^{4}}+\ldots\right]
$$

for $x \gg 1$ to obtain the approximation

$$
\Gamma(c) \approx 1+2 c^{2}
$$


On the other hand, $\Gamma(c) \rightarrow c \sqrt{\pi / 2}$ as $c \rightarrow \infty$. The resulting average wavespeed (4.15) is plotted as a function of $\epsilon$ and various unperturbed speeds $c$ in figure 2(b). The corresponding critical period $\epsilon_{0}$ for propagation failure is shown in figure $3\left(\right.$ a) with $a \epsilon_{0} \rightarrow \sqrt{2 / \pi}$ as $c \rightarrow \infty$. It can be seen that the behavior is very similar to the exponential case. However, the critical period $\epsilon_{0}$ is no longer linear in the threshold $\alpha$, see figure $3(\mathrm{~b})$. The solution of equation (4.2) for the waveform $U(\xi)$ is now

$$
U(\xi)=\frac{1}{2}\left[\operatorname{erfc}(\xi / \sqrt{2})-\exp \left(\frac{\xi}{c}+\frac{1}{2 c^{2}}\right) \operatorname{erfc}\left(\frac{\xi}{\sqrt{2}}+\frac{1}{\sqrt{2} c}\right)\right]
$$

such that

$$
\alpha=\frac{1}{2}\left[1-\exp \left(\frac{1}{2 c^{2}}\right) \operatorname{erfc}\left(\frac{1}{\sqrt{2} c}\right)\right]
$$

\subsection{Higher-order corrections}

So far in our analysis of piecewise linear dynamics we have only considered the lowest-order contribution to $\Phi_{1}(\phi / \epsilon)$ in equation (4.10). We now look at higher-order corrections in $\epsilon$ and show that there are major qualitative differences between the exponential and Gaussian weight distributions. Substituting $A(x)=\mathrm{e}^{i x}$ into equation (4.10) and integrating by parts gives

$$
\Phi_{1}(\phi / \epsilon)=\frac{1}{K(c)} \frac{c \mathrm{e}^{i \phi / \epsilon}}{c+i \epsilon} \int_{0}^{\infty} w(x)\left[\mathrm{e}^{-x / c}-\mathrm{e}^{-i x / \epsilon}\right] d x
$$

which we rewrite in the form

$$
\Phi_{1}(\phi / \epsilon)=\frac{1}{K(c)} \frac{c}{c+i \epsilon}\left[I(c)-I_{+}(\epsilon)+i I_{-}(\epsilon)\right] e^{i \phi / \epsilon}
$$

with

$$
\begin{gathered}
I(c)=\int_{0}^{\infty} w(x) \mathrm{e}^{-x / c} d x \\
I_{+}(\epsilon)=\int_{0}^{\infty} w(x) \cos (x / \epsilon) d x, \quad I_{-}(\epsilon)=\int_{0}^{\infty} w(x) \sin (x / \epsilon) d x
\end{gathered}
$$

Therefore, if $A(x)=a \sin (x)$ then

$$
\Phi_{1}(\phi / \epsilon)=a \widehat{\Gamma}(c, \epsilon) \sin (\phi / \epsilon+\eta)
$$

so that the average wavespeed is

$$
\bar{c}=\sqrt{c^{2}-\epsilon^{2} a^{2} \widehat{\Gamma}(c, \epsilon)^{2}}
$$

with

$$
\widehat{\Gamma}(c, \epsilon)=\frac{1}{K(c)} \frac{c}{\sqrt{c^{2}+\epsilon^{2}}} \sqrt{\left(I(c)-I_{+}(\epsilon)\right)^{2}+I_{-}(\epsilon)^{2}}
$$


Since $\widehat{\Gamma}(c, \epsilon) \rightarrow \Gamma(c) \equiv I(c) / K(c)$ in the limit $\epsilon \rightarrow 0$, the inclusion of higher-order corrections have a negligible effect when $\epsilon$ or $c$ is sufficiently small. In particular the critical period $\epsilon_{0}$ is linear in $c$ for slow waves irrespective of the choice of $w$. However, for faster waves and larger values of $\epsilon$, the approximation $\widehat{\Gamma}(c, \epsilon) \approx \Gamma(c)$ breaks down and major differences emerge between the exponential and Gaussian weight distributions. This is illustrated in figure 4 where the average wavespeed $\bar{c}$ of equation (4.38) is plotted as a function of $\epsilon$ for various unperturbed wavespeeds $c$. Also shown for comparison are the corresponding results obtained using equation (4.15), that is, without the inclusion of higher-order corrections to $\Phi_{1}$. It can be seen that in the case of the exponential distribution these higher-order terms increase the critical period $\epsilon_{0}$ for wave propagation failure, that is, wave propagation failure is suppressed. On the other hand, $\epsilon_{0}$ is reduced for a Guassian distribution so that wave propagation failure is enhanced. Moreover, in the latter case these effects persist down to much smaller values of $\epsilon$.
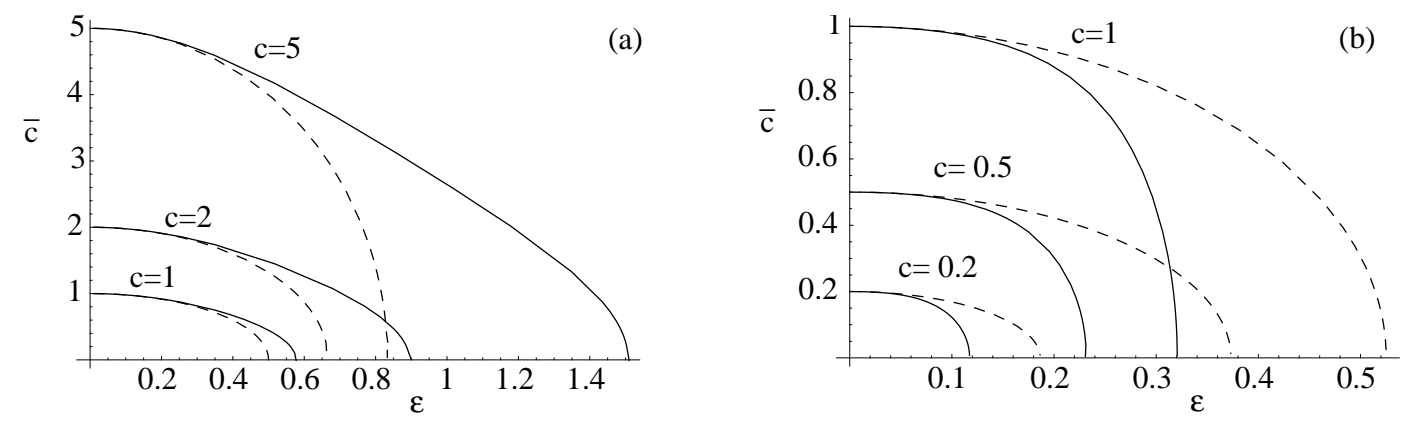

Figure 4: Average wavespeed $\bar{c}$ satisfying equation (4.38) is plotted as a function of $\epsilon$ for a sinusoidal modulation of unit amplitude. (a) Exponential weight distribution and (b) Gaussian weight distribution. The corresponding results in the absence of higher-order corrections to $\Phi_{1}$ are shown as dashed curves.

The difference between the two cases can be understood in terms of the behavior of the functions $I_{ \pm}(\epsilon)$ defined by equation (4.36). First, substituting the exponential distribution (3.4) into (4.36) gives

$$
I_{+}(\epsilon)=\frac{1}{2} \frac{\epsilon^{2}}{\epsilon^{2}+1}, \quad I_{-}(\epsilon)=\frac{1}{2} \frac{\epsilon}{\epsilon^{2}+1}
$$

so that

$$
\widehat{\Gamma}(c, \epsilon)=\frac{\Gamma(c)}{\sqrt{1+\epsilon^{2}}}
$$

The factor $\left(1+\epsilon^{2}\right)^{-1 / 2}$ leads to the partial suppression of wave propagation failure shown in figure 4(a). It is also clear that for this example $\widehat{\Gamma}(c, \epsilon)$ can be expanded as a power series in $\epsilon$ and thus incorporated into higher-order contributions to the perturbation expansion 
of equation (3.8). A very different situation holds, however, for the Gaussian distribution (4.23) where

$$
I_{+}(\epsilon)=\frac{1}{\sqrt{2 \pi}} \int_{0}^{\infty} \mathrm{e}^{-x^{2} / 2} \cos (x / \epsilon) d x=\frac{1}{2} \exp \left(-\frac{1}{2 \epsilon^{2}}\right)
$$

and

$$
\begin{aligned}
I_{-}(\epsilon) & =\frac{1}{\sqrt{2 \pi}} \int_{0}^{\infty} \mathrm{e}^{-x^{2} / 2} \sin (x / \epsilon) d x \\
& =\frac{1}{2} \exp \left(-\frac{1}{2 \epsilon^{2}}\right) \operatorname{Im} \operatorname{erfc}\left(-\frac{i \epsilon}{\sqrt{2}}\right)=\frac{\epsilon}{\sqrt{2 \pi}}\left[1+\epsilon^{2}+3 \epsilon^{4}+\ldots\right]
\end{aligned}
$$

Now $I_{+}(\epsilon)$ is exponentially small in the sense of asymptotics and thus cannot be absorbed into higher-order terms arising in the perturbation expansion of equation (3.8). Although $I_{+}(\epsilon)$ is negligible for very small $\epsilon$ it contributes dramatically at larger values so that the average wavespeed drops off more rapidly than expected as shown in figure 4(b).

It is interesting to compare our results to those of Keener for reaction-diffusion models of excitable chemical media $[10,11]$. In these systems exponentially small terms do not occur for piecewise linear dynamics but do occur in the case of smooth nonlinearities. This implies that there are major qualitative differences between the two types of nonlinearity. On the other hand, this distinction is blurred in the case of excitable neural media since exponentially small terms can also arise for piecewise nonlinearities for certain types of weight distribution. Recall that in the case of an exponential distribution, the integrodifferential equation (3.1) is equivalent to the reaction-diffusion system (3.5) and (3.6). Therefore, it is not surprising that in this case there are no exponentially small terms for piecewise dynamics. On the other hand, a Gaussian distribution introduces an additional smoothing that does generate such terms.

\subsection{Smooth nonlinearities}

The analysis of wave propagation failure in the presence of smooth nonlinearities (finite $\beta$ ) is considerably more complicated than the piecewise case $(\beta \rightarrow \infty)$ since, in order to obtain a reasonable approximation for the average wavespeed, it is necessary to develop the perturbation analysis of equation (3.8) to $\mathcal{O}\left(\epsilon^{2}\right)$ rather than $\mathcal{O}(\epsilon)$. (Higher-order averaging was also necessary in the models analyzed by Keener $[10,11])$. We sketch the basic steps in the analysis.

Let us return to the general first-order equation (3.20) for $\Phi_{1}(\phi / \epsilon)$. Taking $A(x)=\mathrm{e}^{i x}$ and using Fourier transforms,

$$
K \Phi_{1}\left(\frac{\phi}{\epsilon}\right)=-\frac{i}{\epsilon} \mathrm{e}^{i \phi / \epsilon} \int_{-\infty}^{\infty} \widetilde{w}(q) \widetilde{V}^{*}(q) \widetilde{f(U)}\left(q+\epsilon^{-1}\right) \frac{d q}{2 \pi}
$$


where $^{*}$ denotes complex conjugate and

$$
\widetilde{w}(q)=\int_{-\infty}^{\infty} \mathrm{e}^{i q x} w(x) d x
$$

In the case $f(U(\xi))=\Theta(-\xi)$,

$$
\widetilde{V}(q)=\frac{1}{i q-c^{-1}}, \quad \widetilde{f(U)}(q)=\frac{1}{i q+0^{+}}
$$

so that

$$
K \Phi_{1}\left(\frac{\phi}{\epsilon}\right)=\frac{\mathrm{e}^{i \phi / \epsilon}}{\epsilon} \int_{-\infty}^{\infty} w(x)\left[\int_{-\infty}^{\infty} \frac{\mathrm{e}^{i q x}}{\left(q+\epsilon^{-1}-i 0^{+}\right)(q-i / c)} \frac{d q}{2 i \pi}\right] d x
$$

Closing the contour in the upper-half (lower-half) complex $q$-plane for $x>0(x<0)$ we find that there are contributions from the poles at $q=-\epsilon^{-1}+i 0^{+}$and $q=i / c$ such that

$$
K \Phi_{1}\left(\frac{\phi}{\epsilon}\right)=\frac{\mathrm{e}^{i \phi / \epsilon}}{\epsilon} \frac{1}{\epsilon^{-1}+i c^{-1}} \int_{0}^{\infty} w(x)\left[\mathrm{e}^{-x / c}-\mathrm{e}^{-i x / \epsilon}\right] d x
$$

which recovers equation (4.33). It is clear from this analysis that $\Phi_{1}(\phi / \epsilon)$ is of the form (4.12) for all $w$ and there is no problem approximating the wavespeed according to equation (3.21). However, in the case of smooth nonlinearities the Fourier transforms $\widetilde{V}(q)$ and $\widetilde{f(U)}(q)$ are no longer given by simple poles and in general $\Phi_{1}$ will consist of exponentially small terms. It follows that $\Phi_{1}$ may be less significant than the $\mathcal{O}\left(\epsilon^{2}\right)$ terms ignored in the perturbation expansion of (3.8).

In light of the above discussion, take

$$
\phi^{\prime}(\tau)=c+\epsilon \phi_{1}^{\prime}(\tau)+\epsilon^{2} \phi_{2}^{\prime}(\tau)+\ldots
$$

and carry out a perturbation expansion of equation $(3.8)$ to $\mathcal{O}\left(\epsilon^{2}\right)$ :

$$
-\frac{\partial u_{m}(\xi, \tau)}{\partial \tau}+\mathcal{L} u_{m}(\xi, \tau)=-\phi_{m}^{\prime}(\tau) U^{\prime}(\xi)+h_{m}(\xi, \phi / \epsilon)
$$

where for $m=1,2$

$$
\begin{aligned}
h_{1}(\xi, \phi / \epsilon) & =-\int_{-\infty}^{\infty} A\left(\left[\xi^{\prime}+\phi\right] / \epsilon\right)\left[w^{\prime}\left(\xi-\xi^{\prime}\right) f\left(U\left(\xi^{\prime}\right)\right)-w\left(\xi-\xi^{\prime}\right) \frac{d f\left(U\left(\xi^{\prime}\right)\right)}{d \xi^{\prime}}\right] d \xi^{\prime} \quad(4.51) \\
h_{2}(\xi, \phi / \epsilon) & =-\phi_{1}^{\prime} u_{1}^{\prime}(\xi)-\frac{1}{2} \int_{-\infty}^{\infty} w\left(\xi-\xi^{\prime}\right) f^{\prime \prime}\left(U\left(\xi^{\prime}\right)\right)\left[u_{1}\left(\xi^{\prime}\right)\right]^{2} d \xi^{\prime} \\
& -\int_{-\infty}^{\infty} A\left(\left[\xi^{\prime}+\phi\right] / \epsilon\right) w^{\prime}\left(\xi-\xi^{\prime}\right) f^{\prime}\left(U\left(\xi^{\prime}\right)\right) u_{1}\left(\xi^{\prime}\right) d \xi^{\prime} \\
& +\int_{-\infty}^{\infty} A\left(\left[\xi^{\prime}+\phi\right] / \epsilon\right) w\left(\xi-\xi^{\prime}\right)\left[f^{\prime}\left(U\left(\xi^{\prime}\right)\right) u_{1}^{\prime}\left(\xi^{\prime}\right)+f^{\prime \prime}\left(U\left(\xi^{\prime}\right)\right) U^{\prime}\left(\xi^{\prime}\right) u_{1}\left(\xi^{\prime}\right) d \xi^{\prime}\right] d \xi^{\prime}
\end{aligned}
$$


Equation (4.50) then has a bounded solution if and only if

$$
K \phi_{m}^{\prime}(\tau)=\int_{-\infty}^{\infty} V(\xi) h_{m}(\xi, \phi / \epsilon) d \xi
$$

The solvability condition for $m=1$ yields exponentially small terms as argued above. In order to evaluate the solvability condition for $m=2$, it is first necessary to determine $u_{1}(\xi, \phi / \epsilon)$ from equation (4.50). The crucial observation is that if $A(x)$ is a sinusoid then $u_{1}(\xi, \phi / \epsilon)$ will include terms proportional to $\sin (\phi / \epsilon)$ and $\cos (\phi / \epsilon)$. Consequently, substituting for $u_{1}$ in equation (4.52) will generate terms of the form $\sin ^{2}(\phi / \epsilon)$ and $\cos ^{2}(\phi / \epsilon)$ due to the quadratic term in $u_{1}$ on the right-hand side of (4.52). Using the identities $2 \sin ^{2}(x)=1-\cos (2 x)$ and $2 \cos ^{2}(x)=1+\cos (2 x)$, it follows that there will be an $\epsilon$ independent contribution to $\phi_{2}^{\prime}$. Thus for smooth nonlinearities, equation (3.19) takes the form

$$
\frac{d \phi}{d \tau}=c+\epsilon^{2} A_{2}(c)+B_{2}\left(c, \frac{\phi}{\epsilon}\right)
$$

where $A_{2}$ is independent of $\epsilon$ and $B_{2}$ is exponentially small in $\epsilon$. An analogous result holds for reaction-diffusion equations $[10,11]$.

\section{Discussion}

In this paper we have shown that even a weak heterogeneity can have a dramatic effect on wave propagation in an excitable neural medium. If the wavefront solution of the corresponding homogenized system is sufficiently slow then propagation failure can occur. Our work was motivated in part by the observation that there is an approximate periodic microstructure in primary visual cortex that could support a heterogeneous periodic modulation in the long-range connections between neurons. Whether or not such a heterogeneity actually exists within cortex remains to be seen. It might be more appropriate to model the cortex as a disordered medium in which there are random spatial fluctuations in an underlying homogeneous medium. It would be of interest to investigate the effects of structural disorder on wave propagation. Another important extension of our current work is to that of two-dimensional waves, which are obviously more realistic from the viewpoint of cortical modeling. 


\section{References}

[1] H. R. Wilson and J. D. Cowan. Excitatory and inhibitory interactions in localized populations of model neurons. Biophysical J., 12:1-24, 1972.

[2] H. R. Wilson and J. D. Cowan. A mathematical theory of the functional dynamics of cortical and thalamic nervous tissue. Kybernetik, 13:55-80, 1973.

[3] G. B. Ermentrout. Neural networks as spatial pattern forming systems. Rep. Prog. Phys., 61:353-430, 1998.

[4] G. B. Ermentrout and J. D. Cowan. A mathematical theory of visual hallucination patterns. Biol. Cybernetics, 34:137-150, 1979.

[5] P. C. Bressloff, J. D. Cowan, M. Golubitsky, P. J. Thomas, and M. Wiener. Geometrical hallucinations, Euclidean symmetry and the functional architectureal of striate cortex. Accepted for publication in Phil. Trans. B, 2000.

[6] G. B. Ermentrout and J. B. Mcleod. Existence and uniqueness of travelling waves for a neural network. Proc. Roy. Soc. Edin. A, 123:461-478, 1993.

[7] M. A. P. Idiart and L. F. Abbott. Propagation of excitation in neural network models. Network, 4:285-294, 1993.

[8] R. D. Chervin, P. A. Pierce, and B. W. Connors. Propagation of excitation in neural network models. J. Neurophysiol., 60:1695-1713, 1988.

[9] D. Golomb and Y. Amitai. Propagating neuronal discharges in neocortical slices: Computational and experimental study. J. Neurophysiol., 78:1199-1211, 1997.

[10] J. P. Keener. Propagation of waves in an excitable medium with discrete release sites. SIAM J. Appl. Math., 2000.

[11] J. P. Keener. Homogenization and propagation in the bistable equation. Physica D, 136:1-17, 2000.

[12] K. Obermayer and G. G. Blasdel. Geometry of orientation and ocular dominance columns in monkey striate cortex. J. Neurosci., 13:4114-4129, 2000.

[13] N. V. Swindale. The development of topography in visual cortex: a review of models. Network, 7:161-247, 1996. 
[14] D. H. Hubel and T. N. Wiesel. Functional architecture of macaque monkey visual cortex. Proc. Roy. Soc. B, 198:1-59, 1977.

[15] D. H. Hubel and T. N. Wiesel. Receptive fields, binocular interaction and functional architecture in the cat's visual cortex. J. Physiol. Lond., 160:106-154, 1962.

[16] D. H. Hubel and T. N. Wiesel. Receptive fields and functional architecture of monkey striate cortex. J. Physiol. Lond., 195:215-243, 1968.

[17] D. H. Hubel and T. N. Wiesel. Sequence regularity and geometry of orientation columns in the monkey striate cortex. J. Comput. Neurol., 158:295-306, 1974.

[18] G. G. Blasdel and G. Salama. Voltage sensitive dyes reveal a modular organization in monkey striate cortex. Nature, 321:579-585, 1986.

[19] T. Bonhoeffer and A. Grinvald. Orientation columns in cat are organized in pinwheel like patterns. Nature, 364:166-146, 1991.

[20] G. G. Blasdel. Orientation selectivity, preference, and continuity in monkey striate cortex. J. Neurosci., 12:3139-3161, 1992.

[21] M. S. Livingstone and D. H. Hubel. Specificity of intrinsic connections in primate primary visual cortex. J. Neurosci., 4:2830-2835, 1984.

[22] R. B. H. Tootell, S. L. Hamilton, M. S. Silverman, and E. Switkes. Functional anatomy of macaque striate cortex. J. Neurosci., 8:1500-1568, 1988.

[23] A. G. Leventhal, K. G. Thompson, D. Liu, L. M. Neumann, and S. J. Ault. Form and color are not segregated in mokey striate cortex. Invest. Opthamol. Vis. Sci., 34:813, 1993.

[24] D. H. Hubel and T. N. Wiesel. Uniformity of monkey striate cortex: A parallel relationship between field size, scatter, and magnification factor. J. Comp. Neurol., 158:295-306, 1974.

[25] A. Michalski, G.I. Gerstein, J. Czarkowska, and R. Tarnecki. Interactions between cat striate cortex neurons. Exp Brain Res, 53:97-107, 1983.

[26] R. J. Douglas, C. Koch, M. Mahowald, K. A. C. Martin, and H. H. Suarez. Recurrent excitation in neocortical circuits. Science, 269:981-985, 1995.

[27] J. D. Hirsch and C. D. Gilbert. Synaptic physiology of horizontal connections in the cat's visual cortex. J. Neurosci., 11:1800-1809, 1991. 
[28] C. D. Gilbert. Horizontal integration and cortical dynamics. Neuron, 9:1-13, 1992.

[29] R. Malach, Y. Amir, M. Harel, and A. Grinvald. Relationship between intrinsic connections and functional architecture revealed by optical imaging and in vivo targeted biocytin injections in primate striate cortex. Proc. Natl. Acad. Sci., 90:10469-10473, 1993.

[30] W. H. Bosking, Y. Zhang, B. Schofield, and D. Fitzpatrick. Orientation selectivity and the arrangement of horizontal connections in tree shrew striate cortex. J. Neurosci., 17:2112-2127, 1997.

[31] T. Yoshioka, G. G. Blasdel, J. B. Levitt, and J. S. Lund. Relation between patterns of intrinsic lateral connectivity, ocular dominance, and cytochrome oxidase-reactive regions in macaque monkey striate cortex. Cerebral Cortex, 6:297-310, 1996.

[32] U. Eysel. Turning a corner in vision research. Nature, 399:641-644, 1999.

[33] K. S. Rockland and J. Lund. Intrinsic laminar lattice connections in primate visual cortex. J. Comp. Neurol., 216:303-318, 1983.

[34] C. D. Gilbert and T. N. Wiesel. Clustered intrinsic connections in cat visual cortex. J. Neurosci., 3:1116-1133, 1983.

[35] B. A. McGuire, C. D. Gilbert andP. K. Rivlin, and T. N. Wiesel. Targets of horizontal connections in macaque primary visual cortex. J. Comp. Neurol., 305:370-392, 1991.

[36] J Guckenheimer and P J Holmes. Nonlinear Oscillations, Dynamical Systems and Bifurcations of Vector Fields. Springer-Verlag, New York, 1983.

[37] J. P. Keener. Principles of Applied Mathematics: Transformation and Approximation. Addison-Wesley, Reading, MA (2nd edition), 1988. 